\title{
Duplex Real-Time PCR Assay for Clinical Differentiation of Onchocerca lupi and Onchocerca volvulus
}

\author{
Marcos de Almeida, ${ }^{\star}$ Fernanda S. Nascimento, Blaine A. Mathison, Henry Bishop, Richard S. Bradbury, Vitaliano A. Cama, and \\ Alexandre J. da Silva \\ Division of Parasitic Diseases and Malaria, Center for Global Health, Centers for Disease Control and Prevention, Atlanta, Georgia
}

\begin{abstract}
In the United States and Europe, human onchocerciasis is a rare disease caused by zoonotic or anthropophilic parasites in the genus Onchocerca. The zoonotic species identified in focal areas of Europe and United States is Onchocerca lupi, and Onchocerca volvulus, the anthroponotic species, may be found among people who had lived in endemic areas of Africa, the Arabian Peninsula, or Latin America. Onchocerciasis due to O. lupi is an emergent parasitic disease, with limited diagnostic methods, in addition to the lack of information on its biology, transmission, and epidemiology. Cutaneous nodules are the disease's most prevalent manifestation but lack diagnostic specificity. To address the diagnosis of onchocerciasis at reference laboratories, we developed a duplex TaqMan real-time PCR (qPCR) method, targeting the cytochrome oxidase subunit I locus which has species-specific probes to identify and differentiate $O$. lupi from 0 . volvulus. We determined the performance of the duplex with a panel of 45 samples: 11 positives for $O$. lupi, six for O. volvulus, five samples with negative results for Onchocerca spp., and 23 non-Onchocerca nematodes. The duplex qPCR correctly detected 10 of 11 O. Iupi-and six of six O. volvulus-positive specimens. The new duplex assay allowed the simultaneous detection and discrimination of $O$. lupi and $O$. volvulus in clinical specimens, expediting and facilitating the clinical diagnosis of $O$. lupi in non-endemic settings where the disease is an infrequent finding.
\end{abstract}

\section{INTRODUCTION}

Human onchocerciasis is caused by zoonotic and anthropophilic species parasites in the genus Onchocerca, including Onchocerca lupi which is a zoonotic species. It was first described in 1967 from the eye of a wolf in Georgia. Since then, O. Iupi infections have been reported in at least 90 dog and 22 human cases in focal areas of Europe and North America. ${ }^{1,2}$ Other veterinary Onchocerca species such as Onchocerca gutturosa, Onchocerca cervicalis, Onchocerca jakutensis, and Onchocerca dewittei japonica have also been infrequently implicated in human onchocerciasis. ${ }^{3-7}$ Onchocerca volvulus, the anthropophilic species transmitted by black flies of the genus Simulium, causes human onchocerciasis, also known as river blindness. An estimated 21 million people worldwide are infected, ${ }^{8}$ with approximately 205 million people ${ }^{9}$ living at risk of infection in focal endemic areas of Africa, the Arabian Peninsula, and Latin America. ${ }^{10-12}$ Human migration has been the main source of epidemics throughout, facilitating emergence of new infections and changing the distribution, severity, and frequency of known infections. ${ }^{13}$ Given the great increase in international and adventure travel over the past four decades, for epidemiological and clinical diagnostic perspectives, reference laboratories may have to consider the use of standardized methods that allow simultaneous detection and differentiation of these parasites.

In humans, O. Iupi has particular characteristics not commonly observed in other zoonotic filarial infections, such as the presence of multiple worms in a single patient including gravid females. ${ }^{1}$ Cutaneous/subcutaneous nodules are the most common clinical manifestations of $O$. lupi infection; however, cervical spine involvement has been associated with a few cases. ${ }^{1,2,14}$ Spinal onchocercoma manifestations are not observed in 0 . volvulus,

\footnotetext{
* Address correspondence to Marcos de Almeida, Division of Parasitic Diseases and Malaria, Center for Global Health, Centers for Disease Control and Prevention, 1600 Clifton Rd., MS D-64, Atlanta, GA 30329. E-mail: bnz0@cdc.gov
}

where the primary manifestations are severe itching, depigmentation or fibrosis of the skin, and progressive ocular lesions that could lead to partial or total visual impairment. ${ }^{11,15}$ Onchocerca lupi is an emerging parasite, and there is an increasing interest to fully understand its biology, transmission vectors, geographical distribution, epizootiology in dogs, identification of other potential reservoirs, and human epidemiology.

Although serologic methods are used for monitoring $O$. volvulus infection in control and elimination programs, examination of skin snip biopsies is used for the clinical diagnosis of human onchocerciasis. ${ }^{10,16-20}$ Species-level identification of larvae by microscopy is performed only by well-trained staff, requiring appropriate preservation and processing of specimens to ensure that morphologic features are not lost. Adult Onchocerca spp. can be identified by histopathology of nodules, and the species identification relies on features of the multilayered cuticle such as annular ridges (on the external layer) and transverse striae (on the internal layer), as well as size of the worm, appearance of the somatic muscles, size of the lateral chords, and structure of the vagina. ${ }^{10,18-20}$

In comparison with microscopy, molecular techniques are often more sensitive and specific for the detection of positive cases, especially when the clinical specimens have low parasite density, or the morphologic features have been degraded during processing. ${ }^{16,21-23}$ Detection of $O$. volvulus by conventional PCR relies most often on amplification of O-150 locus, a highly polymorphic DNA segment with approximately 4,500 copies per Onchocerca sp. genome. ${ }^{24,25}$ Amplification of 125 ribosomal RNA genes ( $r R N A$ ) and cytochrome oxidase subunit I (COX-I) has also been successfully used for confirmatory detection of Onchocerca species. ${ }^{5,17,26-28}$ Molecular diagnosis of onchocerciasis is increasingly relying on real-time PCR (qPCR) because it is a highly sensitive technique, and is faster than conventional PCR followed by DNA sequence analysis. It also minimizes cross contamination and provides results in a manner which facilitates data analysis. The qPCR assays used for identification of Onchocerca species have shown improved diagnostic performance when compared with conventional PCR methods. ${ }^{23,29-31}$ 
In our laboratory, specimens from suspected cases of onchocerciasis are first examined by microscopy, which is followed by sequencing the products of PCR amplification of the mitochondrial $12 S$ and $16 S$ rRNA loci. However, this methodology is time consuming, and may sometimes provide inconclusive results depending on the specimen tested. To address this limitation and provide a new alternative, we modified a qPCR TaqMan method for O. Iupi. ${ }^{29}$ The modified assay used newly designed genus-specific primers and two species-specific TaqMan probes targeting the COX-I locus, to simultaneously detect and differentiate $O$. Iupi from $O$. volvulus. The results of the duplex qPCR were compared with microscopy and conventional PCR, showing that this assay could expedite the differential diagnosis of onchocerciasis in reference laboratories.

\section{MATERIALS AND METHODS}

Panel of specimens tested. The performance of the $O$. lupi/O. volvulus duplex qPCR assay was evaluated using 45 DNA samples from 11 clinical specimens collected from nine patients positive for $O$. Iupi, adult worms isolated from six clinical cases with positives results for $O$. volvulus, five human tissue biopsies submitted for $O$. lupi diagnosis with negative results, 11 specimens from noninfected people, and 12 from other helminths: Wuchereria bancrofti, Brugia malayi, Dirofilaria immitis, Trichinella nativa, Trichinella spiralis, Trichinella murrelli, Trichinella T6, Anisakis sp., Paragonimus mexicanus, Trichuris sp., Taenia solium, and Halicephalobus gingivalis. In addition, DNA-extracted adult worms previously identified as $O$. Iupi and O. volvulus by microscopy were used as qPCR assay reference controls. Details on the geographic origin and date of collection, microscopic parasite identification, and the type of samples used for DNA extractions are presented in Table 1.

DNA extraction. DNA was extracted using DNeasy Blood and Tissue Kit (Qiagen, Germantown, MD), following the manufacturer's instructions, ${ }^{32,33}$ using either 10-20 mg of human biopsies, $0.2 \mathrm{~mL}$ of blood, or worm sections of $1-10 \mathrm{~mm}$ in length.

Oligonucleotides design. Oligonucleotide primers were designed for conventional PCR, DNA sequence analysis, and qPCR amplification.

Conventional PCR. We designed genus-level primers targeting the mitochondrial $12 S$ and $16 S$ rRNA genes. Primers for the 12S locus Onch12SF/Onch12SR were designed using sequences of O. lupi GenBank (accession no. KC763784), O. volvulus (accession no. AY462920), O. gutturosa (accession no. AY462923), O. jakutensis (accession no. HQ717719), and O. dewittei japonica (accession no. AM779814). The primers Onch16SF/Onch16SR amplified the 16S locus and were based on sequences from O. volvulus (accession no. AY462903) and $O$. gutturosa (accession no. AY462904). These primers were used for our in-house conventional PCR designed to amplify fragments of 270 and 430 bp of the $12 S$ and $16 S$ rRNA, respectively.

TaqMan qPCR. Sequences of COX-I gene of Onchocerca species, including O. lupi (accession nos. JX080028 and HQ207644), O. volvulus (accession no. AM749285), O. gutturosa (accession no. KP760201), O. dewittei japonica (accession no. KP760203), Onchocerca lienalis (accession no. KX853325), Onchocerca fasciata (accession no. JQ316672), Onchocerca flexuosa (accession no. HQ214004), and Onchocerca ochengi (accession no. KX181289), were used as references for the design of primers and probes of the duplex qPCR assay. We designed primers and probes to amplify and detect a fragment of the COX-1 gene previously reported. ${ }^{29}$ Primers ONCOXI-F and ONCOXI-R were designed to be genus specific and amplify products of approximately $135 \mathrm{bp}$. ONCOXI$F$ was designed with two degenerate nucleotides (nt9-R and nt12-Y) to amplify both $O$. lupi and $O$. volvulus. The speciesspecific probes were $O$. lupi OLCOXI-PB and $O$. volvulus OVCOXI-PB, labeled with the FAM (fluorescein-amidite; 6carboxyfluorescein) and VIC (aequorea victoria green fluorescent protein; 2'-chloro-7' phenyl-1,4-dichloro-6-carboxy-fluorescein) fluorophores, respectively. Oligonucleotide and probe sequences are listed in Table 2.

Onchocerca lupi/O. volvulus duplex qPCR assay. The duplex qPCR protocol was optimized as described elsewhere. ${ }^{29}$ The reactions were prepared with $0.25 \mu \mathrm{M}$ of each primer, $0.15 \mu \mathrm{M}$ of each probe, $12.5 \mu \mathrm{L}$ of TaqMan ${ }^{\circledR}$ Universal PCR Master Mix (Applied Biosystems, Waltham, MA), $3 \mu \mathrm{L}$ of DNA template, and sterile water to adjust the volume to $25 \mu \mathrm{L}$. PCR was performed on ABI 7500 real-time PCR system (Applied Biosystems) using the following cycling parameters: $50^{\circ} \mathrm{C}$ for 2 minutes, $95^{\circ} \mathrm{C}$ for 10 minutes, followed by 40 cycles of $95^{\circ} \mathrm{C}$ for 15 seconds and $60^{\circ} \mathrm{C}$ for 1 minute. Fluorescence data were collected at the end of each $60^{\circ} \mathrm{C}$ plateau.

Reproducibility and evaluation of limit of detection (LOD) of O. Iupi/O. volvulus duplex qPCR assay and specimen's quality control. The reproducibility of duplex qPCR was verified by testing all samples at least on two separate runs, on different dates. The LOD was performed in reactions prepared with mixed 0 . lupi/O. volvulus DNA extracted from worms of both species in 10-fold dilutions ranging from 3.0 to $3 \times 10^{-12} \mu \mathrm{g} / \mu \mathrm{L}$. Internal quality control for DNA integrity was based on the SYBR green detection of a fragment of the human $\beta$-actin gene, as previously described. ${ }^{34}$

Conventional PCR and Sanger DNA sequencing analysis. $12 S$ and $16 S$ PCR reactions (12SPCR and 16SPCR) were prepared with $0.2 \mu \mathrm{M}$ each of $16 \mathrm{~S}$ or $12 \mathrm{~S}$ primers, $45 \mu \mathrm{L}$ of Platinum Blue PCR SuperMix mixture (Invitrogen, Waltham, MA), and $3 \mu \mathrm{L}$ of DNA. The total volume of the PCR reactions was 50 $\mu \mathrm{L}$. The amplification protocol was as follows: $95^{\circ} \mathrm{C}$ for 2 minutes, followed by 40 cycles of $95^{\circ} \mathrm{C}$ for 30 seconds, $60^{\circ} \mathrm{C}$ for $30 \mathrm{sec}-$ onds, and $72^{\circ} \mathrm{C}$ for 1 minute, with a final extension of $72^{\circ} \mathrm{C}$ for 5 minutes. The amplicons were purified with StrataPrep PCR purification kit (Stratagene, La Jolla, CA) and subjected to Sanger sequencing using BigDye version 3.1 chemistry (Applied Biosystems) following the manufacturer's instructions. Conventional PCR amplification and cycle sequencing were carried out in a GeneAmp PCR system 9700 thermocycler (Applied Biosystems). Amplification products were visualized by gel electrophoresis.

The amplified reaction mixtures were purified through DyeEx 2.0 Spin Kit (Qiagen) and analyzed on an ABI Prism 3130XL sequence analyzer, with data collection software, V3.0 (Applied Biosystems). The sequences were assembled, edited, and aligned in DNA STAR SeqMan v. 14.0 .0 (88) 422 (DNASTAR Inc., Madison, WI) software.

Next-generation sequencing (NGS). Amplicon deep sequencing was used to obtain DNA sequences from samples that had yielded unreadable Sanger sequences (overlapping peaks in the electropherograms). Those samples were reamplified in triplicates and DNA libraries prepared using NEBNext Ultra II DNA Library Prep Kit (New England Biolabs, Ipswich, MA) as per manufacturer's instructions for $1 \mathrm{ng}$ of DNA input. The libraries were quantified using a Qubit fluorometer 2.0 with dsDNA HS assay kit (Invitrogen-Thermo 
TABLE 1

List of specimens investigated using the O. lupi/O. volvulus real-time PCR duplex assay

\begin{tabular}{|c|c|c|c|c|c|}
\hline Patient number & DNA number & Parasite & Origin, ${ }^{\star}$ year & Sample typeł & Additional information $\ddagger$ \\
\hline 1 & 1 & O. lupi & AZ, 2014 & FFPE & Human nodule biopsy \\
\hline \multirow[t]{2}{*}{2} & 2 & O. lupi & TX/NM/CO, 2014 & FFPE & Human nodule biopsy \\
\hline & 3 & O. Iupi & TX/NM/CO, 2014 & FFPE & Human nodule biopsy \\
\hline 3 & 4 & O. lupi & AZ, 2014 & FFPE & Human nodule biopsy \\
\hline 4 & 5 & O. lupi & NM/CO, 2014 & FFPE & Human nodule biopsy \\
\hline 5 & 6 & O. lupi & AZ/UT, 2014 & FFPE & Human nodule biopsy† \\
\hline 2 & 7 & O. lupi & TX/NM/CO, 2014 & FFPE & Human nodule biopsy† \\
\hline 6 & 8 & O. lupi & NM, 2019 & FFPE & Human nodule biopsy \\
\hline 7 & 9 & O. lupi & NM, 2019 & FFPE & Human nodule biopsy \\
\hline 8 & 10 & O. lupi & $A Z, 2017$ & FFPE & Human nodule biopsy \\
\hline 9 & 11 & O. lupi & TX, 2014 & Worm & - \\
\hline 10 & 12 & O. volvulus & Chad, NA & Worm & Adult $O$. volvulus $\dagger$ \\
\hline 11 & 13 & O. volvulus & Chad, NA & Worm & Adult O. volvulus $\dagger$ \\
\hline 12 & 14 & O. volvulus & Chad, NA & Worm & Adult O. volvulus $\dagger$ \\
\hline 13 & 15 & O. volvulus & DRC, NA & Worm & Adult O. volvulus $\dagger$ \\
\hline 14 & 16 & O. volvulus & Ethiopia, NA & Worm & Adult O. volvulus $\dagger$ \\
\hline 15 & 17 & O. volvulus & S. Sudan, NA & Worm & Adult O. volvulus $\dagger$ \\
\hline 16 & 18 & Negative & AZ, 2015 & Fresh tissue & Human nodule biopsy \\
\hline 17 & 19 & Negative & French Polynesia, 2016 & Blood & Human blood \\
\hline \multirow[t]{3}{*}{18} & 20 & Negative & S. Sudan, 2017 & FFPE & Human nodule biopsy \\
\hline & 21 & Negative & S. Sudan, 2017 & FFPE & Human nodule biopsy \\
\hline & 22 & Negative & S. Sudan, 2017 & FFPE & Human nodule biopsy \\
\hline NA & $23-33$ & Negative human DNA & USA, 2019 & Fresh tissue & Human skin biopsy \\
\hline NA & 34 & Wuchereria bancrofti & CDC collection & FFPE & Filaria, worm $\dagger$ \\
\hline NA & 35 & Brugia malayi & CDC collection & FFPE & Filaria, worm† \\
\hline NA & 36 & Dirofilaria immitis & CDC collection & Fresh tissue & Filaria, adult worm \\
\hline NA & 37 & Trichuris sp. & CDC collection & Fresh tissue & Nematode, adult worm \\
\hline NA & 38 & Trichinella nativa & CDC collection & Fresh tissue & Nematode, larvae \\
\hline NA & 39 & Trichinella spiralis & CDC collection & Fresh tissue & Nematode, larvae \\
\hline NA & 40 & Trichinella murrelli & CDC collection & Fresh tissue & Nematode, larvae \\
\hline NA & 41 & Trichinella T6 & CDC collection & Fresh tissue & Nematode, larvae \\
\hline NA & 42 & Anisakis simplex & CDC collection & Fresh tissue & Nematode, larva \\
\hline NA & 43 & Taenia solium & CDC collection & FFPE & Cestode, tapeworm \\
\hline NA & 44 & Paragonimus mexicanus & CDC collection & FFPE & Trematode, adult worm \\
\hline NA & 45 & Halicephalobus gingivalis & CDC collection & FFPE & Nematode-free living \\
\hline
\end{tabular}

Fisher, Waltham, MA), and the peak sizes were assessed with TapeStation using D1000 DNA Screen Tape (Agilent, Santa Clara, CA). The barcoded libraries were diluted to $4 \mathrm{nM}$, pooled, and 15 PM were loaded in a MiSeq Kit $2 \times 250$ (Illumina, San Diego, CA). The resulting reads were imported into CLC Genomics workbench 11.0 for trimming (quality and adapters) and mapping. The consensus sequences from each of those products were queried in GenBank using the BLASTn tool. We used results which had at least $97 \%$ identity to the sequences previously used for oligonucleotide design.

\section{RESULTS}

Microscopy analysis. The characterization of 22 samples, including 17 suspected cases of onchocerciasis, was performed on the basis of morphological features, that is, the thickness of the cuticle, presence or absence of outer cuticular ridges, and the position of the inner cuticular striae. Based on these characteristics, four worm specimens from three patients were identified as $O$. Iupi, and samples isolated from six cases were identified as $O$. volvulus. Seven Onchocerca adults of unknown species were identified in another six clinical

TABLE 2

Oligonucleotides used in the preparation of the multiplex real-time PCR assay and 16S/12S conventional PCR

\begin{tabular}{llr}
\hline \multicolumn{1}{c}{ Name } & \multicolumn{1}{c}{ Oligonucleotide sequences $\left(5^{\prime}-3^{\prime}\right)$} & Reference sequence \\
\hline ONCOXI-F & TT ATT GGR GGY GGT CCT GGT A & JX080028 \\
ONCOXI-R & TAA TAG CAC CCA ACA AAG AAC C & COX-I (110-131) \\
OLCOXI-PB & FAM - TAG AGT AGA GGG TCA GCC TGA GTT ATC-BHQ1 & JX08028 \\
OVCOX I-PB & HEX - AGG GTT GAA GGT CAA CCA GAA TTG TC- BHQ1 & JX080028 \\
Oncho 12S-F & TIT GAA CTG GAT TAG TAC CCA G & AM749285 \\
Oncho 12S-R & AAA AAT TTA TAA TAG TAA CAC ATG & KC763784 \\
Olupi16SF & GAT GGC ATA AAA GTA GCG TAA GTG A & KC763784 \\
Olupi16SR & AAG ATA AAC CGC TCT GTC TCA CG & COX-I (158-182) \\
\hline
\end{tabular}


samples, and five specimens from three cases were found to be negative for Onchocerca species (Table 3).

Quality control. All the 45 DNA extracts had positive amplification of the $\beta$-actin locus by SYBR Green qPCR, indicating a lack of PCR inhibitors in the extracted DNA. However, sample 4 was of limited availability for the DNA extraction and yields $\beta$-actin gene SYBR Green cycle threshold (Ct) values of 37.5. Compared with the average $\mathrm{Ct}$ values among the other Onchocerca spp.-positive samples $(\leq 23.2)$, the high Ct value of sample 4 was indicative of very low DNA concentration in the sample (Table 3).

Performance of 0 . Iupi/O. volvulus duplex qPCR. The performance of the duplex qPCR assay for the discrimination of $O$. Iupi and $O$. volvulus was verified using the suspected 22 onchocerciasis cases submitted to our laboratory for diagnostic confirmation by microscopy. All clinical samples were also analyzed by conventional PCR and sequencing of the $12 S$ and $16 S$ loci (samples 1-22, Table 1). From the 22 clinical samples, five samples were negative by all tests. From the remaining 17 specimens, 16 were positive by microscopy and PCR: 11 were O. Iupi and six were O. volvulus. One of the 17 specimens (4) was microscopy positive for $O$. lupi but negative by PCR.

The duplex qPCR consistently amplified those 16 positive samples and correctly identified either $O$. lupi or $O$. volvulus. The 11 samples with $O$. lupi had a single FAM fluorescent signal with $\mathrm{Ct}$ values ranging from 18 to 35 . The six samples

TABLE 3

Summary of results obtained from the O. lupi/O. volvulus biplex qPCR assay, conventional PCR, followed by DNA sequencing and microscopy analysis

\begin{tabular}{|c|c|c|c|c|c|c|c|}
\hline \multirow[b]{2}{*}{ Specimen number } & \multirow[b]{2}{*}{ Type } & \multicolumn{3}{|c|}{ Biplex qPCR } & \multicolumn{2}{|c|}{$\begin{array}{l}\text { Conventional PCR followed by DNA } \\
\text { sequencing } †\end{array}$} & \multirow[b]{2}{*}{ Microscopy } \\
\hline & & $\begin{array}{l}\text { O. lupi Ct } \\
\text { value (FAM) }\end{array}$ & $\begin{array}{l}\text { O. volvulus Ct } \\
\text { value (HEX) }\end{array}$ & Internal control Ct value & 12S PCR Sequencing & 16S PCR Sequencing & \\
\hline 1 & FFPE & 29.02 & Negative & 21.6 & O. lupi & O. lupi & O. lupi \\
\hline 2 & FFPE & 30.35 & Negative & 22.2 & O. lupi & O. lupi & O. lupi \\
\hline 3 & FFPE & 33.53 & Negative & 21.9 & O. lupi*† & Weak* $\ddagger$ & O. lupi \\
\hline 4 & Fresh tissue & Negative & Negative & 37.5 & Negative & Negative & O. lupi \\
\hline 5 & FFPE & 28.38 & Negative & 23.2 & O. lupi`† & O. lupi`† & Onchocerca sp. \\
\hline 6 & FFPE & 35.03 & Negative & 21.8 & Weak $^{*} \ddagger$ & O. lupi*† & Onchocerca sp. \\
\hline 7 & FFPE & 30.37 & Negative & 21.5 & O. lupi & O. lupi & Onchocerca sp. \\
\hline 8 & FFPE & 30.43 & Negative & 22.2 & Weak $^{\star} \ddagger$ & O. lupi & Onchocerca sp. \\
\hline 9 & FFPE & 31.37 & Negative & 22.3 & Weak $^{\star} \ddagger$ & O. Iupi & Onchocerca sp. \\
\hline 10 & FFPE & 27.37 & Negative & 21.4 & O. lupi & Weak $^{*} \ddagger$ & Onchocerca sp. \\
\hline 11 & Worm & 18.58 & Negative & 22.3 & O. Iupi & O. Iupi & Onchocerca sp. \\
\hline 12 & Worm & Negative & 25.27 & 20.7 & O. volvulus ${ }^{\star} \dagger$ & O. volvulus ${ }^{\star} \dagger$ & O. volvulus \\
\hline 13 & Worm & Negative & 20.39 & 21.3 & O. volvulus & O. volvulus & O. volvulus \\
\hline 14 & Worm & Negative & 22.22 & 21.0 & O. volvulus & O. volvulus & O. volvulus \\
\hline 15 & Worm & Negative & 20.20 & 21.0 & O. volvulus & O. volvulus & O. volvulus \\
\hline 16 & Worm & Negative & 24.28 & 22.0 & O. volvulus & O. volvulus & O. volvulus \\
\hline 17 & Worm & Negative & 14.99 & 21.9 & O. volvulus & O. volvulus & O. volvulus \\
\hline 18 & Fresh tissue & Negative & Negative & 21.3 & Negative & Negative & Negative \\
\hline 19 & Blood & Negative & Negative & 21.1 & Negative & Negative & Negative \\
\hline 20 & FFPE & Negative & Negative & 22.0 & Negative & Negative & Negative \\
\hline 21 & FFPE & Negative & Negative & 21.8 & Negative & Negative & Negative \\
\hline 22 & FFPE & Negative & Negative & 26.8 & Negative & Negative & Negative \\
\hline 23 & Human DNA & Negative & Negative & 24.8 & NA & NA & NA \\
\hline 24 & Human DNA & Negative & Negative & 22.1 & NA & NA & NA \\
\hline 25 & Human DNA & Negative & Negative & 26.4 & NA & NA & NA \\
\hline 26 & Human DNA & Negative & Negative & 20.6 & NA & NA & NA \\
\hline 27 & Human DNA & Negative & Negative & 24.5 & NA & NA & NA \\
\hline 28 & Human DNA & Negative & Negative & 25.7 & NA & NA & NA \\
\hline 29 & Human DNA & Negative & Negative & 27.9 & NA & NA & NA \\
\hline 30 & Human DNA & Negative & Negative & 25.9 & NA & NA & NA \\
\hline 31 & Human DNA & Negative & Negative & 20.4 & NA & NA & NA \\
\hline 32 & Human DNA & Negative & Negative & 20.9 & NA & NA & NA \\
\hline 33 & Human DNA & Negative & Negative & 26.9 & NA & NA & NA \\
\hline 34 & Wuchereria bancrofti & Negative & Negative & 29.7 & NA & NA & NA \\
\hline 35 & Brugia malayi & Negative & Negative & 34.3 & NA & NA & NA \\
\hline 37 & Dirofilaria immitis & Negative & Negative & 33.8 & NA & NA & NA \\
\hline 37 & Trichuris sp. & Negative & Negative & 30.8 & NA & NA & NA \\
\hline 38 & Trichinella nativa & Negative & Negative & 32.4 & NA & NA & NA \\
\hline 39 & Trichinella spiralis & Negative & Negative & 21.3 & NA & NA & NA \\
\hline 40 & Trichinella murrelli & Negative & Negative & 25.6 & NA & NA & NA \\
\hline 41 & Trichinella T6 & Negative & Negative & 24.3 & NA & NA & NA \\
\hline 42 & Anisakis simplex & Negative & Negative & 20.6 & NA & NA & NA \\
\hline 43 & Taenia solium & Negative & Negative & 31.4 & NA & NA & NA \\
\hline 44 & Paragonimus mexicanus & Negative & Negative & 23.6 & NA & NA & NA \\
\hline 45 & Halicephalobus gingivalis & Negative & Negative & 32.3 & NA & NA & NA \\
\hline
\end{tabular}

FFPE = formalin-fixed paraffin-embedded; O. lupi = Onchocerca lupi; O. volvulus = Onchocerca volvulus; qPCR = real-time PCR. SYBR Green qPCR internal control (Ct values) represents the quality of DNA aliquots.

*DNA aliquots weakly amplified.

† Species identified by using partial Sanger sequencing and/or next-generation sequencing.

† Failed to provide sequencing data. 
positive for $O$. volvulus had a single VIC fluorescent signal, with $\mathrm{Ct}$ values ranging from 15 to 25 . No fluorescent signal was detected in the five negative samples (18-22). The real-time and $12 S$ and $16 S$ conventional PCR failed to amplify specimen 4. The molecular test results were $100 \%$ concordant between the conventional and qPCR, with an overall sensitivity of $91 \%$ (10/11), for O. lupi and $100 \%(n=6)$ for O. volvulus.

The duplex qPCR specificity was $100 \%$, as shown by the negative amplification of human samples and DNA extracted from other helminths (samples 23-45). The LOD of the duplex qPCR was calculated to be $<3 \times 10^{-6} \mathrm{ng} / \mu \mathrm{L}$. Results are summarized in Table 3.

Species-specific identification of $O$. Iupi and $O$. volvulus using conventional PCR followed by DNA sequencing analysis. $12 S$ PCR. Sixteen of the 22 samples tested by microscopy had positive amplification (nine nodule biopsies and seven worms), including five clinical specimens weakly amplified $(3,5,6,8$, and 9$)$. The sequencing data of these amplicons were compared with the GenBank database allowing the identification of seven $O$. lupi cases, including two identified only with partial sequences (specimens 3 and 5 ) and six $O$. volvulus cases. Three amplicons (specimens 6, 8, and 9) failed to provide sequences.

$16 S$ PCR. To verify species identification, the 22 samples were further analyzed at the $16 \mathrm{~S}$ locus. All 16 samples with positive 12S amplification were positive at the $16 \mathrm{~S}$ locus and subjected to DNA sequencing, including five samples with weak amplification products $(3,5,6,10$, and 12$)$. Six samples (12-17) had $>99 \%$ of identity to $O$. volvulus. Of the remaining 10 samples, nine had highly homologous sequences, showing $94 \%$ identity with O. flexuosa and O. lienalis (accession nos. HQ214004 and AY462906), respectively, in the GenBank database. Because GenBank did not have sequences for $O$. Iupi at this locus, we submitted the consensus sequence from specimens 1 and 2 (accession no. KM347895). One sample (specimen 10), failed to provide sequences. In addition, specimen 4 , previously diagnosed as positive for $O$. lupi by microscopy, did not amplify at either the $16 \mathrm{~S}$ or $12 \mathrm{~S}$ loci. The five samples (18-22) previously diagnosed negative for Onchocerca spp. by microscopy were also negative at both $16 \mathrm{~S}$ and $12 \mathrm{~S}$ loci. Results are summarized in Table 3.

Next-generation sequencing for species verification of $12 S$ and $16 S$ amplicons. We used NGS to obtain complete sequences from seven samples that weakly amplified at the $12 \mathrm{~S}$ and/or $16 \mathrm{~S}$ loci. Specimens 3, 5, 6, and 12 provided enough amplified DNA for NGS analysis, whereas amplification of specimens 8,9 , and 10 failed at both loci. The NGS data were generated from mapping sequences with $\times 100$ coverage on average.

12S. The amplicon sequencing of specimen 12 had $99 \%$ similarity with O. volvulus (accession no. AY462920). The sequences from nodule biopsies 3 and 5 were homologues, with $100 \%$ identity with O. Iupi (accession no. KC763784) reference. Specimen 6 did not yield enough NGS reads for analysis.

16S. The consensus sequence of specimen 12 was $>99 \%$ similar to O. volvulus (accession no. AY462903). The sequences of specimens 5 and 6 had $>99 \%$ similarity with O. lupi (accession no. KM347895). Specimen 3 did not yield NGS data.

\section{DISCUSSION}

Human onchocerciasis in non-endemic settings is an infrequent finding, and it has usually been linked to people who lived for extended periods in endemic areas. However, $O$. Iupi has recently emerged in Tunisia, Iran, Greece, Germany, and the United States, areas not endemic for $O$. volvulus. $5,20,22,27,35$ Although the geographic separation of $O$. lupi and O. volvulus is evident, migrant populations or travelers can harbor many infections contributing to introduction, adaptation, and circulation of pathogenic microbes into new geographic areas. Depending on the presence of vectors and the hosts' susceptibility, this factor contributes to the emergence of infectious diseases, increasing the public health implications, rates of transmission, and infectivity. ${ }^{13}$ Therefore, because infections by $O$. lupi may have more severe clinical manifestations than $O$. volvulus, including cervical spine involvement, reference laboratories must provide species-level identification of Onchocerca spp. to properly assist in decisions for medical treatment. In addition, accurate parasite identification provides a better understanding on the distribution and prevalence of the disease, especially in patients who may have an extensive travel history. ${ }^{19,36,37}$

In our laboratory, suspected cases of onchocerciasis are investigated using both microscopy and PCR, followed by DNA sequencing analysis. Because the conventional PCR methods were designed using generic Onchocerca primers, they can theoretically amplify any Onchocerca parasite, and DNA sequence analysis could determine the species as either O. Iupi or O. volvulus. However, as demonstrated in this study, the suboptimal amplification of some specimens may not allow species identification by DNA sequencing. Consequently, we developed a new TaqMan qPCR assay targeting the COX-I locus for the detection and specific identification of $O$. lupi and O. volvulus in clinical specimens. This novel qPCR assay was adapted from the method described by Latrofa et al., ${ }^{29}$ into the duplex assay format using one primer pair and speciesspecific TaqMan probes for O. Iupi and O. volvulus.

Although microscopic analysis is the most common technique used for the diagnosis of infection with O. lupi and O. volvulus, the sensitivity and specificity of microscopy depends on the quality of the specimen and the microscopy skills of the staff tasked with identifying parasites. DNA-based detection methods are more sensitive methods to detect Onchocerca spp. ${ }^{38-40}$; however, the efficiency of these methods (specificity and sensitivity) depends on the quality of the preserved specimen and DNA template, set of oligonucleotides selected, and the size of fragments amplified. For instance, the O-150 locus, the standard target used for the detection of $O$. volvulus in programs for monitoring and elimination of onchocerciasis, has intraspecies variation that can affect the sensitivity of PCR methods. ${ }^{23}$ The rRNA and COX-I genes are multi-copy genes with low rates of polymorphism, providing species-specific results to distinguish Onchocerca spp. by multiplex qPCR assays. ${ }^{21,29,38-41}$

The performance of the new qPCR in this study demonstrated good detection limits and did not cross-react with human DNA or other helminths tested. The results of the qPCR were in agreement with $12 S$ and $16 S$ PCR results and showed 95\% (20/21) agreement with microscopic analysis. It should be noted that the molecular methods missed one case (specimen 4) that was microscopy positive for O. lupi. We attributed the discrepant results to the low concentration of total DNA obtained from the very small amount of clinical sample available for DNA extraction (approximately $1 \mathrm{~mm}$ of tissue). This hypothesis is supported by the weak amplification of this DNA aliquot using the SYBR qPCR (Ct. 37.5). This finding 
highlights the importance of adequate amount and preservation of the specimen to be tested. The analytical sensitivity or LOD of the duplex qPCR was similar to that of single target assays reported in other studies. ${ }^{29,32}$ Although the detection of $O$. Iupi and $O$. volvulus was concordant with PCR and sequencing results, the qPCR allowed the species-specific identification of these parasites, providing faster and simultaneous identification, including in those cases that provided only partial or no sequencing data, or cases identified only to the genus level by microscopy. It should be emphasized that the efficiency of PCR assays may be affected by aforementioned factors. Samples poorly preserved will lead to substandard amplification, possibly because of DNA denaturation, or by the presence of PCR inhibitors which can be found in DNA extracts from FFPE. ${ }^{42}$

\section{CONCLUSION}

The duplex qPCR method allowed the identification of $O$. Iupi while differentiating from $O$. volvulus. This duplex $\mathrm{qPCR}$ TaqMan improved and streamlined the clinical diagnosis of $O$. lupi onchocerciasis in a U.S. reference laboratory, showing advantages over CPCR and DNA sequencing.

Received February 10, 2020. Accepted for publication June 18, 2020. Published online August 3, 2020.

Financial support: This study was supported by the CDC/CGH/DPDM, Atlanta, GA.

Disclaimer: The findings and conclusions in this article are those of the authors and do not necessarily represent the official position of the CDC/the Agency for Toxic Substances and Disease Registry and the U.S. Food and Drug Administration.

Disclosure: Clinical samples were used in accordance with a CDC human subject-approved protocol (IRB no. 5756). Data sharing is not applicable to this article as no datasets were generated or analyzed during the current study in addition to the data presented in Tables 1 and 3 .

Authors' addresses: Marcos de Almeida, Fernanda S. Nascimento, Henry Bishop, Richard S. Bradbury, and Vitaliano A. Cama, Division of Parasitic Diseases and Malaria, Center for Global Health, Centers for Disease Control and Prevention, Atlanta, GA, E-mails: bnz0@cdc.gov or maredalm@yahoo.com, fs_nascimento@yahoo.com.br, hsb2@ cdc.gov, r.bradbury@federation.edu.au, and vec5@cdc.gov. Blaine A. Mathison, Division of Parasitic Diseases and Malaria, Center for Global Health, Centers for Disease Control and Prevention, Atlanta, GA, and ARUP Laboratories (current address), Institute for Clinical and Experimental Pathology, Salt Lake City, UT, E-mail: blaine.mathison@ aruplab.com. Alexandre J. da Silva, Division of Parasitic Diseases and Malaria, Center for Global Health, Centers for Disease Control and Prevention, Atlanta, GA, and Food and Drug Administration (current address), Center for Food Safety and Applied Nutrition (CFSAN), Office of Applied Research and Safety Assessment, Laurel, MD, E-mails: bnz0@cdc.gov or alexandre.dasilva@fda.hhs.gov.

\section{REFERENCES}

1. Cantey PT et al., 2016. The emergence of zoonotic Onchocerca lupi infection in the United States-a case-series. Clin Infect Dis 62: 778-783.

2. Gracio AJ, Richter J, Komnenou AT, Gracio MA, 2015. Onchocerciasis caused by Onchocerca lupi: an emerging zoonotic infection. Systematic review. Parasitol Res 114: 2401-2413.

3. Faisca P, Morales-Hojas R, Alves M, Gomes J, Botelho M, Melo $\mathrm{M}$, Xufre A, 2010. A case of canine ocular onchocercosis in Portugal. Vet Ophthalmol 13: 117-121.

4. Koehsler M, Soleiman A, Aspock H, Auer H, Walochnik J, 2007. Onchocerca jakutensis filariasis in humans. Emerg Infect Dis 13: $1749-1752$.
5. Otranto D, Eberhard ML, 2011. Zoonotic helminths affecting the human eye. Parasit Vectors 4: 41.

6. Uni $S$ et al., 2017. Zoonotic infection with Onchocerca dewittei japonica in an 11-year-old boy in kansai region, western Honshu, Japan. Parasitol Int 66: 593-595.

7. Uni S et al., 2015. New zoonotic cases of Onchocerca dewittei japonica (Nematoda: Onchocercidae) in Honshu, Japan. Parasit Vectors 8: 59

8. World Health Organization, 2018. Progress report on the elimination of human onchocerciasis, 2017-2018. Wkly Epidem Rec 93: 633-648.

9. WHO, 2018. Onchocerciasis - Fact Sheet. Geneva, Switzerland: World Health Organization. https://apps.who.int/iris/bitstream/ handle/10665/275983/WER9347.pdf?ua=1. Accessed December 28, 2018.

10. Takaoka $\mathrm{H}$ et al., 2004. Zoonotic onchocerciasis caused by a parasite from wild boar in Oita, Japan. A comprehensive analysis of morphological characteristics of the worms for its diagnosis. Parasite 11: 285-292.

11. Udall DN, 2007. Recent updates on onchocerciasis: diagnosis and treatment. Clin Infect Dis 44: 53-60.

12. WHO, 2018. Onchocerciasis - River Blindness. Geneva, Switzerland: World Health Organization. Available at: http://www.who.int/ mediacentre/factsheets/fs095/en/. Accessed November 30, 2018.

13. Wilson ME, 1995. Travel and the emergence of infectious diseases. Emerg Infect Dis 1: 39-46.

14. Dudley RWR, Smith C, Dishop M, Mirsky D, Handler MH, Rao S, 2015. A cervical spine mass caused by Onchocercalupi. Lancet 386: 1372.

15. Basanez MG, Pion SD, Churcher TS, Breitling LP, Little MP, Boussinesq M, 2006. River blindness: a success story under threat? PLoS Med 3: e371.

16. Lagatie O, Verheyen A, Nijs E, Van Dorst B, Batsa Debrah L, Debrah A, Supali T, Sartono E, Stuyver LJ, 2018. Evaluation of the diagnostic performance of Onchocerca volvulus linear epitopes in a peptide enzyme-linked immunosorbent assay. Am J Trop Med Hyg 98: 779-785.

17. Vincent JA, Lustigman S, Zhang S, Weil GJ, 2000. A comparison of newer tests for the diagnosis of onchocerciasis. Ann Trop Med Parasitol 94: 253-258.

18. Abegunde AT, Ahuja RM, Okafor NJ, 2016. Doxycycline plus ivermectin versus ivermectin alone for treatment of patients with onchocerciasis. Cochrane Database Syst Rev 2016: CD011146.

19. Biswas A, Yassin MH, 2013. An unexpected cause of eye irritation: a case of zoonotic ocular onchocerciasis. Case Rep Infect Dis 2013: 504749.

20. Eberhard ML, Ostovar GA, Chundu K, Hobohm D, Feiz-Erfan I, Mathison BA, Bishop HS, Cantey PT, 2013. Zoonotic Onchocerca lupi infection in a 22-month-old child in Arizona: first report in the United States and a review of the literature. Am J Trop Med Hyg 88: 601-605.

21. Ishizawa $H$, Kato $S$, Nishimura $H$, Tanaka R, Fukuda $M$, Takaoka H, Tsutsumi Y, 2015. Zoonotic onchocerciasis. Pathol Int 65: 271-273.

22. Otranto D, Dantas-Torres F, Cebeci Z, Yeniad B, Buyukbabani N, Boral OB, Gustinelli A, Mounir T, Mutafchiev Y, Bain O, 2012. Human ocular filariasis: further evidence on the zoonotic role of Onchocerca lupi. Parasit Vectors 5: 84.

23. Mekonnen SA et al., 2017. O-5S quantitative real-time PCR: a new diagnostic tool for laboratory confirmation of human onchocerciasis. Parasit Vectors 10: 451.

24. Meredith SE, Lando G, Gbakima AA, Zimmerman PA, Unnasch TR, 1991. Onchocerca volvulus: application of the polymerase chain reaction to identification and strain differentiation of the parasite. Exp Parasitol 73: 335-344.

25. Meredith SE, Unnasch TR, Karam M, Piessens WF, Wirth DF, 1989. Cloning and characterization of an Onchocerca volvulus specific DNA sequence. Mol Biochem Parasitol 36: 1-10.

26. Hassan HK et al., 2015. Isolation of Onchocerca lupi in dogs and black flies, California, USA. Emerg Infect Dis 21: 789-796.

27. Mowlavi G, Farzbod F, Kheirkhah A, Mobedi I, Bowman DD, Naddaf SR, 2014. Human ocular onchocerciasis caused by Onchocerca lupi (Spirurida, Onchocercidae) in Iran. J Helminthol 88: 250-255. 
28. Toe L, Boatin BA, Adjami A, Back C, Merriweather A, Unnasch TR, 1998. Detection of Onchocerca volvulus infection by O-150 polymerase chain reaction analysis of skin scratches. $J$ Infect Dis 178: 282-285.

29. Latrofa MS, Annoscia G, Colella V, Cavalera MA, Maia C, Martin C, Slapeta J, Otranto D, 2018. A real-time PCR tool for the surveillance of zoonotic Onchocerca lupi in dogs, cats and potential vectors. PLoS Negl Trop Dis 12: e0006402.

30. Lloyd MM, Gilbert R, Taha NT, Weil GJ, Meite A, Kouakou IM, Fischer PU, 2015. Conventional parasitology and DNA-based diagnostic methods for onchocerciasis elimination programmes. Acta Trop 146: 114-118.

31. Thiele EA, Cama VA, Lakwo T, Mekasha S, Abanyie F, Sleshi M, Kebede A, Cantey PT, 2016. Detection of Onchocerca volvulus in skin snips by microscopy and real-time polymerase chain reaction: implications for monitoring and evaluation activities. Am J Trop Med Hyg 94: 906-911.

32. Almeida M, Bishop H, Nascimento FS, Mathison B, Bradbury RS, Silva AD, 2018. Multiplex TaqMan qPCR assay for specific identification of encapsulated Trichinella species prevalent in North America. Mem Inst Oswaldo Cruz 113: e180305.

33. Bhatnagar J, Blau DM, Shieh WJ, Paddock CD, Drew C, Liu L, Jones T, Patel M, Zaki SR, 2012. Molecular detection and typing of dengue viruses from archived tissues of fatal cases by RT-PCR and sequencing: diagnostic and epidemiologic implications. Am J Trop Med Hyg 86: 335-340.

34. de Almeida ME, Koru O, Steurer F, Herwaldt BL, da Silva AJ, 2017. Detection and differentiation of Leishmania spp. in clinical specimens by use of a SYBR green-based real-time PCR assay. J Clin Microbiol 55: 281-290.
35. Otranto D, Dantas-Torres F, Giannelli A, Latrofa MS, Papadopoulos E, Cardoso L, Cortes H, 2013. Zoonotic Onchocerca lupi infection in dogs, Greece and Portugal, 2011-2012. Emerg Infect Dis 19: 2000-2003.

36. Nguyen JC, Murphy ME, Nutman TB, Neafie RC, Maturo S, Burke DS, Turiansky GW, 2005. Cutaneous onchocerciasis in an American traveler. Int J Dermatol 44: 125-128.

37. Sanders CJ, Jaspers CA, 2007. Onchocerciasis or loiasis? Lancet 369: 271-272.

38. Crowe A, Koehler AV, Sheorey H, Tolpinrud A, Gasser RB, 2018. PCR-coupled sequencing achieves specific diagnosis of onchocerciasis in a challenging clinical case, to underpin effective treatment and clinical management. Infect Genet Evol 66: 192-194.

39. Lefoulon $E$ et al., 2015. Shaking the tree: multi-locus sequence typing usurps current onchocercid (filarial nematode) phylogeny. PLoS Negl Trop Dis 9: e0004233.

40. Verocai GG, Nelson KJ, Callahan RT, Wekesa JW, Hassan HK, Hoberg EP, 2018. A cryptic species of Onchocerca (Nematoda: Onchocercidae) in blackflies (Simulium spp.) from southern California, USA. Parasit Vectors 11: 547.

41. Casiraghi M, Anderson TJ, Bandi C, Bazzocchi C, Genchi C, 2001. A phylogenetic analysis of filarial nematodes: comparison with the phylogeny of Wolbachia endosymbionts. Parasitology 122: 93-103.

42. Dietrich D, Uhl B, Sailer V, Holmes EE, Jung M, Meller S, Kristiansen G, 2013. Improved PCR performance using template DNA from formalin-fixed and paraffin-embedded tissues by overcoming PCR inhibition. PLoS One 8: e77771. 